\title{
ALGUNOS ANTECEDENTES MEDIEVALES DE LA SEMÁNTICA DE J. KATZ
}

ANGEL MuñoZ

UNIVERSIDAD DE ZULIA

\section{Introduccion}

Desde la primera página, Katz plantea el problema - que estudiará desde distintos puntos de vista a lo largo de toda la obra-,'1 de la necesidad de no detenerse en una lógica de nivel sintáctico, y de pasar al plano de la lógica semántica, si queremos demostrar la validez de muchos argumentos que, de otro modo, quedarían sin explicación consecuencial. Es decir, que se dan argumentos cuya validez no depende de ninguna regla lógica, y cuya justificación está exclusivamente en las relaciones de significado de los componentes de premisa y conclusión. Y lo mismo se diga, a nivel de proposiciones, cuya analiticidad, contradictoriedad, etc., hay que buscar muchas veces, necesariamente, en una explicación semántica.

Lo que nos indica que, del mismo modo que la insuficiencia del cálculo proposicional para justificar un buen número de argumentos, obligó al lógico al estudio del cálculo de predicados, éste presenta también una serie de limitaciones que sólo pueden ser salvadas con una teoría o lógica semántica que aumenta el número de formas lógicas y de reglas -semánticas- de inferencia. El ejemplo claro de lo dicho podría ser el que el propio Katz da en el cap. 4 de su obra:

$$
\frac{\text { Juan es soltero }}{\text { Juan es varón }{ }^{2}}
$$

que, desde un punto de vista sintáctico funcional, sólo es justificable con la solución de Carnap, introduciendo como premisa adicional lo que Katz llama un postulado de significado, de la forma

$$
(x)(S x \supset V x)
$$

1 Katz, J., Teoria semántica, Madrid, 1979. (En adelante las referencias a esta obra se citarán solamente con el nombre del autor.)

2 Katz, 4.128 y 4.129. 
Pero no nos adelantemos; se trataba solamente de establecer el punto de partida de Katz.

Sin embargo, no parece que haya sido este autor el primero en sentir la necesidad de una justificación semántica de ciertos argumentos. Hallamos vestigios de esto ya - al menos vestigios_ en las obras de la lógica medieval que - como se sabe- es eminentemente semántica.

Prescindamos ahora de las críticas que se han hecho frecuentemente a la lógica medieval por sus interminables discusiones en torno, por ejemplo, a los sofismas y falacias; críticas éstas que manifiestan desconocer la profundidad y riqueza de los aportes que el análisis de tales argumentos aportaron a la lógica. Prescindamos de eso y notemos simplemente que muchas de tales falacias estudiadas por los terministas, no son sino otros casos análogos a los que propone Jerrold Katz, cuya solución se da con base, precisamente, en razones más semánticas que sintácticas. Véase, por ejemplo, la siguiente falacia que recoge y estudia Alberto de Sajonia: ${ }^{3}$

\begin{tabular}{l} 
Todo can corre \\
Una constelación es el can \\
\hline Una constelación corre
\end{tabular}

O ésta, muy paralela a otra presentada por el propio. Katz:

Lo que está en el vaso es licor

Un color está en el vaso

Un color es licor

La solución de éstas y otras falacias viene dada por los terministas fundamentalmente con base en la semántica: el que el término medio del primer silogismo no sea uno solo sino dos, no se puede establecer sino atendiendo a su significado.

Otras falacias están basadas en la ambigüedad debida a que una oración del lenguaje natural corresponde en su significado a más de una proposición, como por ejemplo,

\section{Oigo vencer a los troyanos}

Digo a Ayax puede que el ejército troyano venza ${ }^{5}$

3 Alberto de Sajonia, Perutilis Logica, n. 1484. Las referencias a Alberto de Sajonia son de su principal obra lógica Perutilis Logica, cuya edición preparé y que publicará próximamente el Instituto de Investigaciones Filosoficas de la Universidad Nacional Autónoma de México.

4 P. L., n. 1484; compárese con Katz (4): "Tengo un dolor en el pie, mi pie está dentro de mi zapato, luego tengo un dolor en mi zapato."

5 P. L., n. 1498. 
$\mathrm{Y}$ así, se dan falacias por ambigüedad como

Los que veo morder veo que muerden

Veo morder los perros

Veo que los perros muerden ${ }^{6}$

(tanto las dos oraciones citadas independientemente como la premisa menor del paralogismo, son semánticamente ambiguas).

Todo ello, por no citar coincidencias mayores, casi hasta literales, como el ejemplo de Alberto de Sajonia

Tú compraste la carne cocida?

que parece haber inspirado el paralogismo de Katz

Hoy como lo que compré en la tienda la semana pasada Compré un pescado crudo en la tienda la semana pasada

Hoy como un pescado crudo ${ }^{8}$

Pero repasemos algunos de los conceptos sobre los que Katz fundamenta sus teorías y reflexiones, y veamos de compararlo con sus similares: medievales.

Unicamente habrá que señalar el hecho de que el significado en los terministas y en Katz no son referencialmente paralelos, ya que, si para los primeros los términos apuntan directamente al objeto, para el segundo los constituyentes apuntan a los conceptos; teniendo esto en cuenta, veamos algunos de los puntos de la teoría de Katz.

\section{La sinonimia}

$\mathrm{Y}$ comencemos con la explicación que hace sobre la sinonimia. Ésta: tiene un precedente en la similaridad semántica, concebida como la comunidad de un marcador semántico, representativo de un determinado. concepto, en dos -o más- lecturas de dos constituyentes.

El caso límite de esta similaridad sería la sinonimia, que se da cuan-do dos constituyentes tienen en común no ya un marcador, sino una lectura o -en el caso de sinonimia plena- todas las lecturas. Con baseen la sinonimia se podría definir la analiticidad, y de ahí el entrañe.

Pero Katz no llama la atención sobre el hecho de que dos términos.

6 P. L., n. 1499.

7 P. L., n. 612 .

8 Katz (5). 
se llaman en verdad sinónimos sólo cuando ambos se implican mutuamente; es decir, si cada uno de ellos está incluido en el otro, no siendo suficiente el que la inclusión se dé solamente en un sentido. No es quizá del todo la misma advertencia de Alberto de Sajonia, pero hemos de señalar la distinción que hace al respecto. Para el medieval dos términos son sinónimos si significan lo mismo según la misma razón; pero no todos los términos tales son sinónimos, sino aquellos que, además, significan del mismo modo, de tal manera que no esté impedida la predicación mutua del uno respecto del otro. Por eso "hombre" y "hombres" no son sinónimos, puesto que uno no puede predicarse del otro; pero sí lo son "piedra" y "guijarro", aunque sean de distinto género.9 Nótese el paralelismo entre el requisito de Katz — que se impliquen los términos mutuamente- y el de Alberto de Sajonia - que se puedan predicar mutuamente.

Katz no admite que los nombres propios sean sinónimos, pues siendo según él los nombres signos de conceptos, los nombres propios no tienen significado; ${ }^{10}$ este obstáculo no existe para los medievales; traigo esto a colación por el hecho de que el ejemplo que citaré está puesto con base en nombres propios sinónimos; pero esto puede ser circunstancial y se podría haber dado el ejemplo con nombres comunes sinónimos, lo que no haría cambiar el punto de vista medieval.

Teniendo esto en cuenta, Alberto de Sajonia señala abiertamente algo a lo que Katz no alude: que la deducción hecha con base en términos plenamente sinónimos es inútil, no es tal deducción, precisamente —dice Alberto de Sajonia - porque no prueba nada; como en el ejemplo aludido:

$$
\frac{\text { Marco corre }}{\text { Tulio corre }}
$$

(en el supuesto de que previamente se conoce que Marco y Tulio es la misma persona), y ello debido a la sinonimia plena, en la que también parecen haberse adelantado los medievales a Katz. ${ }^{11}$

\section{Entrañe}

La noción de sinonimia nos sugiere la de entrañe. No es que Katz defina ésta en función de la primera: hablo de sugerencia, no de relación.

La relación estaría entre las nociones de sinonimia y de inclusión. (Hemos dicho que Katz exige para la sinonimia la inclusión mutua de

9 P. L., nn. 1278 y $1282-4$.

10 Katz, p. 506 ss.

11 P. L., n. 1616. 
los términos sinónimos.) Si todos los marcadores semánticos en una lectura de un término están también en una lectura de otro, el sentido del primero está semánticamente incluido en el sentido del otro. Y si esto se da con todas las lecturas, tendríamos el caso de la inclusión plena.

Según esto, es claro que la inclusión - como la sinonimia- se da entre los términos de una proposición (no entre proposiciones). Pero si ampliáramos este concepto a la relación entre dos proposiciones, nos estaríamos acercando al concepto de entrañe semántico: con base en esta relación, una proposición se deduce lógicamente de otra, pero no en virtud de alguna ley lógica; es decir, sin pasos intermedios que lleven de la premisa a la conclusión, y sin razón, por consiguiente, para apelar a dicha ley lógica. De modo que la justificación de entrañe depende de que la forma lógica de la conclusión forma parte de la forma lógica de la premisa; pero una forma lógica semántica; es decir, que el entrañe se basa en que las relaciones de significado que se dan entre los componentes de la conclusión forman parte de las relaciones de significado que se dan entre los componentes de la premisa. ( $Y$ así, no hay pasos que lleven progresivamente de la premisa a la conclusión, y por consiguiente no hay motivo para apelar a leyes lógicas.) Por ejemplo,

$$
\frac{\text { Juan es soltero }}{\text { Juan es varón }} \quad \frac{\mathrm{Sj}}{\mathrm{Vj}}
$$

Y ésta es para Katz la razón de que tal deducción se considere válida, y no esta otra:

$$
\frac{\text { Juan es banquero }}{\text { Juan es mezquino }} \quad \frac{\mathbf{B j}}{\mathbf{M j}}
$$

El querer explicar esto obligó a Carnap a introducir lo que Katz llama los "postulados de significado", como

$$
(x)(S x \supset V x)
$$

de modo que, en realidad, el argumento sería

$$
\begin{aligned}
& S j \\
& (x)(S x \supset V x)
\end{aligned}
$$

Para Katz esto es un "postulado intuitivo" que no soluciona nada, si no se recurre a una explicación semántica, ya que no se ve por qué no se pueda aplicar un postulado similar que hiciera válido el argumento 
del banquero mezquino. La única razón no puede ser sino la relación semántica que hay entre "soltero" y "varón", que no la hay entre "banquero" y "mezquino". Además, es claro que si se puede garantizar la validez de la última argumentación - la que incluye el "postulado de intuición"--, ésta no es la misma inicial que constaba de una sola premisa. Por tanto, hemos de concluir que o las relaciones lógicas no son suficientes para deducir, o que las relaciones semánticas pertenecen al número de las relaciones deductivas.

De ahí que haya que buscar la justificación de inferencias -es decir el entrañe-, al menos de algunas, en función de las relaciones semánticas: así como el cálculo de predicados introduce sobre el proposicional el análisis de la proposición - que el proposicional no hace-, análisis que facilitará la justificación de muchas inferencias injustificables en términos de cálculo proposicional, la semántica introduce sobre el funcional el análisis interno de los términos, análisis que el cálculo funcional no hace, y que justificará inferencias como la dicha, injustificables, como se ha visto, en términos del cálculo de predicados. ${ }^{12}$

De uno $u$ otro modo ésta parece ser la solución dada por los medievales. Unas veces con soluciones - y hasta ejemplos_ similares a las que hemos leído en Carnap-Katz, basándose, en el fondo, en la significación de los términos. Como cuando nos dice Alberto de Sajonia que "de la parte subjetiva a su todo universal, la consecuencia es válida", como en

$\frac{\text { Sócrates es hombre }}{\text { Sócrates es animal }{ }^{13}}$

cuyo "postulado semántico intuitivo" para justificarlo, bien podría ser el de que un término común es el todo universal respecto a sus inferiores, que son sus partes subjetivas. ${ }^{14}$ Otros casos similares serian también - de la parte en cuanto al modo, a su todo-.

Un hombre blanco corre

Un hombre corre

o

$\frac{\text { Sócrates es hombre blanco }}{\text { Sócrates es hombre }}$

Son todas ellas consecuencias que los escolásticos llaman "dialécticas"

12 Katz, cap. 4.5 passim, y cap. 6.2 passim.

13 P. L., n. 1302-4.

14 P. L., n. 1289.

15 P. L., n. 1339. 
o "materiales", es decir, basadas en la semántica, distinguiéndolas de las formales ( $\mathrm{y}$ en general se podría decir esto de todos los loci dialectici).

Otras veces esta solución semántica medieval se estudia en tipos concretos de proposición que parecen obligar más a esa solución de carácter semántico, como en el caso de las proposiciones exclusivas:

\section{Solamente un hombre corre}

Solamente un animal corre ${ }^{16}$

que, de nuevo, nos recuerda el ejemplo de Carnap-Katz.

Pero el caso más frecuente, y más claro, es el que se refiere al estudio de las proposiciones y de las inferencias con base en la suposición de los términos. Y recordemos a este respecto que la suposición no es sino la significación concreta que, hic et nunc, tiene un término. Es decir: el término, al tener significación, significa a varios indistintamente; pero al tener suposición tiene una significación más completa, en el sentido de que significa de una manera distinta y precisa; un diccionario - en el sentido común de la palabra, no en el técnico de Katz- sería una colección de términos con significado, pero sin suposición; al estar en la proposición, el término tiene además suposición, que hace restricción de los significados de un término, consiguiéndose, entre otras cosas, evitar la equivocidad del mismo.

Aclaremos en qué consisten algunos de los diferentes tipos de suposición, en concreto -a los que se hará alusión aquí--, la suposición confusa distributiva, suposición determinada, y suposición discreta; y lo haremos siguiendo al mismo Alberto de Sajonia en su muy precisa Perutilis.

Suposición confusa distributiva se da cuando el término se toma en lugar de todos y cada uno - copulativamente_ de los individuos significados por el término; por ejemplo, al decir "todo alumno está inscrito", "alumno" supone confusa y distributivamente, porque significa tanto como "este alumno está inscrito y ese alumno está inscrito y aquel alumno está inscrito, y ..." y así con cada uno de los alumnos. ${ }^{17}$ En la suposición determinada, el término está en lugar de uno cualquiera de entre todos los individuos - tomados disyuntivamente- significados por el término; por ejemplo, el término "alumno" en la proposición "un alumno está becado", que equivale a "este alumno está becado, o ese alumno está becado, o aquel alumno está becado, o ..." y así con cada uno de los alumnos; nótese que si en el caso de la suposición confusa 
distributiva se trataba de todos los alumnos (conjunta $\rightarrow$ copulativamente- todos los alumnos), en la determinada se trata de un alumno -al menos un alumno-, tomado de entre la enumeración de todos los alumnos, aunque no sabemos con exactitud cuál es ese uno. ${ }^{18}$ Por su parte, la suposición discreta se da cuando el término está en lugar de un solo individuo, pero ahora ya un individuo totalmente determinado, como cuando se dice "este alumno es el más brillante".18 Añadamos, para el caso que vamos a estudiar a continuación, que un relativo de identidad, como por ejemplo "distinto", hace que el término que le sigue tenga suposición confusa distributiva. ${ }^{20}$

Según esto, consecuencias como

Sócrates de un hombre es distinto

Sócrates es distinto de un hombre

no son válidas, pues en la premisa y en la conclusión el término debe tener el mismo tipo de suposición ( $y$ no olvidemos que la suposición de un término es su significación concreta hic et nunc); y en la premisa el término "hombre" supone determinadamente (esto es: "Sócrates de este hombre - por ejemplo, Platón- es distinto, o Sócrates de ese hombre es distinto, o Sócrates de aquel hombre es distinto, o...", lo cual es verdad), y en la conclusión el mismo término supone confusa y distributivamente (esto es: "Sócrates es distinto de este hombre, y Sócrates es distinto de ese hombre, y Sócrates...", lo cual, por otra parte, es falso). ${ }^{21}$

O esta otra consecuencia, más similar a la de Carnap-Katz:

$\frac{\text { Sócrates es distinto de Platón }}{\text { Sócrates es distinto de un hombre }}$

Para justificar la invalidez de esta inferencia, Alberto de Sajonia nos recuerda el principio -al que pudiéramos tachar si se quiere, como al de Carnap, de "intuitivo"- de que "de la negación del inferior a la negación del superior, la consecuencia no es válida". Pero es curioso notar que hay otro elemento, el elemento semántico, que juega papel importante en ella: y es que "Platón" supone discretamente (en lugar de uno concreto) y "hombre" confusa y distributivamente. Y por eso mismo —por su significación supositiva_, sí es válida la inferencia:

\footnotetext{
18 P. L., nn. 432 ss.

19 P. L., n. 428.

20 P. L., n. 420.

21 P. L., n. 486.
} 


$\frac{\text { Sócrates es distinto de Platón }}{\text { Sócrates de un hombre es distinto }{ }^{22}}$

Aunque parezca redundancia impertinente, recordemos el caso citado más arriba como calcado por Katz, y cuya explicación viene dada por la apclación (un caso especial de la suposición en el predicado): es falsa la proposición

\section{Tú compraste la carne cocida}

siendo, sin embargo, verdadera esta otra:

\section{La carne cocida tú (la) compraste ${ }^{23}$}

Dejando ya de lado el aspecto de la suposición, hay aún otro, de carácter semántico también, que influye en la validez de la inferencia y que parece nos autoriza a catalogar ciertas referencias medievales como paralelas a la solución semántica de Katz. Me refiero a lo que los medievales llaman condiciones o causas de verdad de una proposición.

Aclaremos previamente que llaman causa de verdad a aquello que es suficiente para que una proposición sea verdadera; por ejemplo, para que sea verdadera la proposición

\section{Un hombre corre}

basta que Pedro corra, basta que Juan corra, etc.; y así, que Juan corra es causa de verdad de la proposición dada. Pero no es causa de verdad, como es claro, de la proposición

\section{Todo hombre corre ${ }^{24}$}

Aclarado esto, Alberto de Sajonia nos dice que "cuando hay dos proposiciones que se dan de tal modo que todas las causas de verdad de una, por ejemplo $A$, son causas de verdad de la otra, por ejemplo $B, y$ no al contrario, entonces la que tiene más causas de verdad se sigue de la que tiene menos, y no al contrario".25

Apliquemos esto al ejemplo de Carnap-Katz. Sean las proposiciones

22 P. L., n. 485.

23 Katz (5) y P. L., n. 612.

24 P. L., n. 687.

25 P. L., nn. 1000-4. 

A
Juan es varón
B
Juan es soltero

Es claro que un análisis - aunque no exhaustivo- de tales proposiciones nos daría, respectivamente, las siguientes causas de verdad:

A tendría como causa de verdad que

- Juan es ser vivo, animado, racional, masculino, no-casado

- Juan es ser vivo, animado, racional, masculino, casado

- Juan es ser vivo, animado, racional, masculino, viudo

- Juan es ser vivo, animado, racional, masculino, divorciado

es decir (al menos) cuatro causas de verdad. $\mathrm{X}$

B tendría como causa de verdad que

- Juan es un ser vivo, animado, racional, masculino, no-casado

pero no las otras tres; es decir (en nuestro análisis) una sola causa de verdad.

Por tanto, la proposición A tiene más causas de verdad que la proposición B. Y según lo dicho por Alberto de Sajonia, de la proposición B, se sigue la proposición A; es decir,

B $\quad$ Juan es soltero

Sin embargo, la justificación de la validez de esta inferencia parece asemejarse más que al postulado intuitivo de Carnap, a la solución semántica de Katz, con base en los marcadores semánticos de "soltero" y de "varón". Con lo que tendríamos aquí un clarísimo antecedente de la lógica semántica de Katz.

E incluso esta solución es aplicable a los casos que estudiábamos un poco más arriba, basados en la suposición, pues el que las causas de verdad sean distintas es

o porque los términos de ambas proposiciones son distintos

o porque los términos suponen de distinto modo

o porque la cópula es distinta ${ }^{28}$

Asimismo, no parece haber necesidad de postulado intuitivo, sino que 26 P. L., n. 1000-6. 
se trata de nuevo de la solución semántica de Katz, cuando la inferencia va de un correlativo al otro, respecto del verbo "ser", es decir, cuando de tales relativos se predica la existencia; y entendiendo relativo no en sentido gramatical, sino predicamental. ${ }^{27}$ (Es claro que se trata de relación asimétrica.) Son relativos en este sentido términos como "mayor" y "menor", "doble" y "mitad", "jefe" y "súbdito", "amo" y "esclavo". Ejemplos de tales inferencias pueden ser

$$
\begin{aligned}
& \text { Hay un amo } \\
& \hline \text { Hay un esclavo } \\
& \text { Hay el doble } \\
& \hline \text { Hay la mitad }{ }^{28}
\end{aligned}
$$

\section{Opacidad}

Vayamos, por último, al caso de la opacidad. Un contexto es opaco, nos dice Katz, si falla la sustitutividad de idénticos. Dada, por ejemplo, la verdad de la identidad

La muchacha que vive encima de Juan = La muchacha más fea del mundo

(identidad simple, contingente), a pesar de todo, no es válida la inferencia

$\frac{\text { Pedro quiere casarse con la muchacha que vive encima de Juan }}{\text { Pedro quiere casarse con la muchacha más fea del mundo }}$

en donde parece claro que se da opacidad.

Analizando dicho argumento desde el punto de vista de los terministas, tenemos que

Muchacha que vive encima de Juan

tiene suposición discreta, es decir, tal término supone en lugar de un objeto - persona en este caso- bien definido y concreto, imposible de

27 La comparación ahora no se hace con lo que Katz llama relativo, que viene siendo más bien un sentido comparativo. Sigo refiriéndome al argumento propuesto por Katz (4.128) y (4.129).

28 P. L., n. 1443.

29 Katz (6.64) al (6.68). 
confundir con otro. Sin embargo, en la conclusión, se da suposición confusa y distributiva; veámoslo:

Lo que nos dice la conclusión es

Pedro quiere casarse con la muchacha que es la más fea de las muchachas del mundo.

Ahora bien: los superlativos o comparativos de superioridad -como "más fea" - hacen que el término que les sigue — "muchacha" en nues. tro caso- tenga suposición confusa y distributiva..$^{30}$ Por tener este tipo de suposición, lo que en verdad quiere decir la conclusión es

Pedro quiere casarse con la muchacha más fea de estas dos

y Pedro quiere casarse con la muchacha más fea de estas tres

y Pedro quiere casarse con la muchacha más fea de estas cuatro

y etc. ...

lo cual es falso, ya que cada una de las proposiciones copuladas, o al menos todas menos una, es falsa, lo que hace falsa a toda la copulativa. Que cada una de las copuladas sea falsa es claro si atendemos a la explicación que hace Alberto de Sajonia, de que en ellas los numerales hay que entenderlos copulativamente, es decir, como un todo, en contraposición a la copulativa usada disyuntivamente. ${ }^{31}$ Pues bien: en "Pedro quiere casarse con la muchacha más fea de estas tres", si ha de tomarse el numeral copulativamente, parece obvio que la proposición es falsa. ${ }^{32}$

De modo pues que, siendo la premisa verdadera y la conclusión falsa, la inferencia es inválida. $Y$, por otro lado, como el término "muchacha" supone de modo diferente en la premisa y en la conclusión, debemos concluir de nuevo la invalidez de la deducción.

A este respecto ya nos advierte Alberto de Sajonia ante ciertos argumentos falaces; y creo que tal advertencia es también aplicable al argumento Carnap-Katz. Nos dice que, aunque un término se verifique

30 P. L., nn. 495 ss.

31 P. L., n. 499.

32 Alberto de Sajonia hace la distinción - que no hemos encontrado en otros medievales_, respecto a la expresión copulativa, de que hay copulativas con sentido copulativo o colectivo, y copulativas con sentido disyuntivo, según que los copulados copulados se tomen o no como formando un solo todo, como es el caso, respectivamente, del sujeto de la proposición "la materia y la forma son un compuesto" o de "una y otra vez comiste pan" (Perutilis, nin. 517 y 518). Pues bien: en "Pedro quiere casarse con la muchacha más fea de estas tres", como el numeral hay que tomarlo copulativamente, la proposición parece falsa, puesto que el sentido sería que "Pedro quiere casarse con la muchacha que tuviera la fealdad sumatoria de la fealdad de estas tres muchachas". 
de otro, no necesariamente el primero se dice de un tercero del mismo modo que se decía del segundo. ${ }^{33}$

Parece ser que ante la solución de Frege al problema de la opacidad, se pone sobre el tapete la acción al respecto de los verbos que podríamos llamar "verbos de inteligencia y voluntad" (creo, sé, dudo, quiero, etc.), y que parecen coincidir con aquellos verbos de los que Alberto de Sajonia dice que su acción recae, a la vez, sobre

- una expresión sustantiva

- una expresión adjetiva unida a la anterior

- el propio nexo que une a ambas ${ }^{34}$

Prescinciendo de otras consideraciones, para no entrar en la polémica de Katz y Frege, señalemos algunos de los casos de inferencias inválidas con base en estos verbos, señalados por Alberto de Sajonia.

En primer lugar 'tenemos casos que pudiéramos llamar opacos debido a la apelación de los términos afectados por este tipo de verbos. Un predicado se dice que apela su forma, si es verificable, en una proposición de presente, del demostrativo que señala al objeto en lugar del cual supone el sujeto de la proposición. Así, en la proposición

El hombre es animal

el predicado "animal" apela su forma, es decir, es verificable del sujeto de la proposición

Esto es animal

en donde "esto" supone en lugar de lo que supone "hombre" en la proposición inicial. ${ }^{35}$ Pues bien: en los casos de proposiciones con los verbos que nos ocupan, la expresión adjetiva que les sigue, no sólo apela su forma, sino también la complexión con la expresión sustantiva; pero si precede al verbo, apela sólo su forma.

Supongamos que Juan conoce a Pedro, y Pedro está sentado, pero Juan no sabe que Pedro está sentado; en este caso, la proposición

\section{A Pedro sedente, Juan (lo) conoce}

es verdadera, según lo que hemos supuesto, pues su sentido es

33 P. L., n. 1589.

34 P. L., n. 622.

35 P. L., nn. 607-8. 


\section{Esto conoce Juan}

señalando con "esto" a Pedro que, por caso, está sentado. Y la proposición, así, es verdadera. Pero en el caso de

$$
\text { Juan conoce a Pedro sedente }
$$

en donde "sedente" no sólo apela su forma sino también su complexión con "Pedro", el sentido es

$$
\text { Juan conoce que Pedro está sentado }
$$

que, por hipótesis, es falso. ${ }^{\mathbf{3 6}}$

Aplicando este planteamiento al caso de la fea vecina de Juan, de la que nos habla Katz, ${ }^{37}$ encontramos un cierto parecido. No pretendo decir que sea el mismo caso de opacidad que estudiamos; pero sí hay una cierta similaridad; tanto en

como en

Pedro quiere desposar a la muchacha vecina de Juan

Pedro quiere desposar a la muchacha más fea,

en ambos casos, tanto "vecina de Juan" como "más fea" apelan su forma y la conexión con la expresión sustantiva "muchacha", y en ese caso tenemos el caso propuesto por Katz de que en la primera proposición Pedro quiere a la muchacha precisamente en cuanto vecina de Juan, y en el segundo precisamente en cuanto más fea (que no tendría que coincidir con la que es precisamente la vecina de Juan, a menos que fueran -aunque sólo sea contingentemente- idénticas).

La clave para resolver estos casos está en si se conoce o no la identidad entre los términos de sustitución. Así, sean las proposiciones

\section{A Pedro busca al Presidente del Consejo de Facultad y \\ B Pedro busca al Decano de la Facultad.}

Si Pedro conoce previamente la identidad

El Presidente del Consejo de Facultad = El Decano de la Facultad, 36 P. L., n. 622.

87 Katz (6.67) y (6.68). 
las partes subrayadas en las proposiciones A y $\mathbf{B}$ son puramente referenciales, y ambas proposiciones son verdaderas, si la primera lo es.

En el citado texto de Alberto de Sajonia se señala expresamente el desconocimiento previo de la identidad al caso: he aquí el texto:

Si tú conoces a Sócrates, y Sócrates está viniendo, y tú no sabes que él viene, esta proposición es verdadera: "a Sócrates viniente tú conoces", y sin embargo esta otra es falsa: "tú conocés a Sócrates que viene".

En la segunda proposición, la expresión adjetiva "que viene" apela su forma, y la conexión con el sustantivo "Sócrates", es decir, que la proposición estaría afirmando que tú conoces la identidad.

$$
\text { Sócrates }=\text { el hombre que viene }
$$

lo cual, en el caso propuesto, no es verdad, con lo que la segunda proposición "tú conoces a Sócrates que viene" sería verdadera, precisamente porque la expresión adjetiva "que viene" apela no sólo su forma, sino también la conexión con "Sócrates".

Por lo mismo, la inferencia de la definición a lo definido (o al contrario), cuando se utilizan estos verbos, no es válida si la definición y lo definido son el predicado de las proposiciones; como aquí:

Sócrates ignora que Platón es animal racional Sócrates ignora que Platón es hombre ${ }^{38}$

Sin pretender pues restar méritos a la tesis de Katz, ni mucho menos atribuir toda la originalidad de la misma a los lógicos medievales, sí parece que habremos de concluir que ya éstos se habían adelantado, sentando -al menos_ las bases de la semántica de Jerrold Katz. 